\title{
Trends and Differentials in Knowledge, Ever Use, Current Use, and Future Intended Use of Contraceptives in Rural Bangladesh: Evidence from Three Surveys
}

\author{
RUhul Amin, A. G. MARIAM and RASHID FARUQEE*
}

\begin{abstract}
The study examines the trends and differentials in knowledge, ever use, current use, and future intended use of contraceptives in rural Bangladesh. Using data from three national-level surveys between 1968 and 1977, the study finds that there had been an increase in contraceptive knowledge, ever use, current use, and future intended use over the years. Although estimated logit regression models indicated that more educated women and women having higher parity were significantly more likely to adopt or to intend to adopt contraceptives in the future, other socio-economic indicators such as regional development or non-agricultural occupations did not lead to higher contraceptive use or future intended use of contraceptives. Somewhat similar levels of differences in ever, current, and future intended use of contraceptives among socio-economic groups persisted over the years. Implications of these findings are discussed in the paper.
\end{abstract}

\section{INTRODUCTION}

Evidence indicates that the pace of diffusion of fertility control can be accelerated in high-fertility societies by introducing advanced contraceptives and family-planning programmes. ${ }^{1}$ On the other hand, despite a record of more than a decade of national-level family-planning programmes, there appears to be little perceptible progress in the acceptance of fertility-control measures in some highfertility societies. Freedman (1979) suggested that this low level of family-planning response is due to official failure in setting up proper administrative, communication, and transportation networks necessary to successfully implement such programmes. Others have alternatively suggested that cultural barriers and low levels of development have operated as obstacles to family-planning progress in these societies. ${ }^{2}$

There is ample evidence suggesting that awareness of and access to contraceptives can greatly shorten the time span for the acceptance of fertility regulation

*Dr Amin and Dr Mariam are Research Scientists at the Morgan State University, Baltimore (Md, U.S.A.), while Dr Faruqee is Senior Economist at the World Bank, Washington, D.C., U.S.A.

${ }^{1}$ See, for example, Knodel and Debavalya (1978), Tsui and Bogue (1978), Teachman, Bogue, Londono, and Hogan (1979), and Mauldin and Berelson (1978).

${ }^{2}$ See Demeney (1975), Coale (1973), and Kirk (1972). 
independent of a nation's level of socio-economic development. ${ }^{3}$ Entwisle, Hermalin, and Kamnuansilpa (1984) as well as Knodel, Havanon, and Pramualratana (1984) have also observed that availability of contraceptives accelerates familylimitation practices by removing socio-economic barriers to the use of familyplanning services and by meeting contraceptive needs. However, the initial experience of certain countries with fertility regulation appears to suggest a lack of demand for fertility control even as modern contraceptives were made available nation-wide.

The analysis presented in this paper attempts to assess this apparent discrepancy by focusing upon socio-economic differences and impact of family-planning programmes on knowledge, use, and future intended use of contraceptives in a society with a low level of development. Analysis of socio-economic differences and their consequences should enable us to increase our understanding of the processes leading both to an increased contraceptive use and to the factors accelerating such use. The data for this study are drawn from three major surveys conducted between 1968 and 1977 in Bangladesh. During this period, there has also been a gradual expansion in family-planning programmes in Bangladesh without a corresponding significant socio-economic development. ${ }^{4}$

This paper has two major objectives. Firstly, it sets forth some hypotheses about differences in knowledge, use, and future intended use of family-planning methods in rural Bangladesh, and secondly, it examines some of these hypotheses in the context of socio-economic trends and differences. The study employs logit analysis in estimating net effects of socio-economic and demographic factors. ${ }^{5}$

\section{FAMILY PLANNING IN BANGLADESH}

Family-planning programmes and family-limitation practices are a relatively recent phenomenon in Bangladesh. ${ }^{6}$ Although a nation-wide family-planning programme had been in existence for about a decade by the mid-1970s, no significant impact of such programmes on fertility or fertility control appeared to be

${ }^{3}$ See, in particular, Tsui and Bogue (1978), Freedman and Berelson (1976), Ravenholt and Chao (1974), and Knodel (1977).

${ }^{4}$ See Arthur and McNicoll (1978) and Demeney (1975) for a detailed discussion in this area.

${ }^{5}$ With our dichotomous dependent variable, the constant-variance assumption of the general linear model is violated and ordinary-least-square estimates are rendered inefficient. Likewise, because dichotomous variables can be non-normal in their distribution, the assumption of a normal error-term and the usual test of significance for least-square techniques are inappropriate. Logit regression technique deals with all these problems. See Goodman (1972).

${ }^{6}$ See Arthur and McNicoll (1978) and Zaidi (1970). These investigators found that contraception or family planning was unknown in Bangladesh prior to the introduction of national family-planning programme. 
evident. ${ }^{7}$ This general lag in programme impact on fertility or fertility control may be expected in view of the recency of family-planning programmes and the persistence of a high-fertility culture in which women with larger families contracept more than those with smaller families, thus masking the effect of contraceptive use on fertility. ${ }^{8}$ Nevertheless, the increasing population pressure and expanding familyplanning programmes were expected to lead to gradual increases in fertility regulation and contraceptive use. Such gradual increases may result more from increased awareness of and access to modern contraceptives brought about by familyplanning programmes than by socio-economic changes, although such changes are likely to facilitate use and future intended use of contraceptives. We hypothesize that knowledge and use of contraceptives might be higher among those who were more exposed to "modern influences". We also hypothesize that contraceptive use might result from demographic pressure measured in terms of number of surviving children. The following analysis will test these hypotheses by examining data from rural Bangladesh for the period between 1968 and 1977.

\section{DATA AND METHODS OF ANALYSIS}

The data for the current analysis came from three sample surveys: Impact of Family Planning Survey (IFPS) of 1968, World Fertility Survey (WFS) for Bangladesh (1975), and Rural Fertility and Poverty Survey of 1977 (RFPS), conducted by the Bangladesh Institute of Development Studies (BIDS). The IFPS was a nationally representative retrospective survey akin to the WFS in content and quality. However, RFPS data were collected from four major regions in Bangladesh. Although not nationally representative, the RFPS data also contained information on fertility and family planning comparable to the other two surveys. In this paper, we shall only utilize data on "currently married" women from the IFPS $(N=2,522)$, WFS $(N=4,117)$, and RFPS $(N=1,247)$. Since the RFPS data were from rural areas, they are compared with corresponding data from rural samples in the IFPS and WFS.

${ }^{7}$ See Amin, Mariam, and Faruqee (1985), Amin and Faruqee (1980), Sirageldin, Norris, and Ahmad (1975a), and Sirageldin, Hossain, and Cain (1975b). These studies did not find any evidence of fertility transition in Bangladesh.

${ }^{8}$ If both users and non-users of contraceptives are equally fertile, contraceptive users will end up with smaller families than those of non-users. But it is often difficult to demonstrate this proposition empirically using data from developing countries because of implicit temporal ordering not typically measured in sample surveys of fertility behaviour. In the event of confusion about when contraception began and how consistently it was used, a positive correlation between current or ever use of contraceptives and fertility may be obtained. This occurs because the decision to use contraceptives is based in part on the number of living children at the time of use. Thus, not only the association between contraceptive use and fertility is in the wrong direction, but also the flow of causality is in;both directions (between fertility and contraception) rather than in one direction (from contraception to fertility). 
Trends and differentials in knowledge, use, and future intended use of contraceptives are relevant for the assessment of the impact and prospects of family. planning programmes. The focus on their determinants will, therefore, be central to the current analysis. In the initial analysis, we focused upon contraceptive use, knowledge of family-planning methods, ideal family size, and intent for future contraceptive use by cross-tabulating them with various independent variables associated with fertility regulation in less developed countries. Subsequently, we assessed the net contribution of each of the independent variables in the explanation of ever, current, and future intended uses of contraceptives by logit regression. ${ }^{9}$ Comparable KAP and other socio-demographic data were collected by the three surveys, as the following sample items indicate:

\section{Ideal Family Size}

IFPS - In your opinion, what is the appropriate desired number of children for your whole life?

WFS - In your opinion, what is the appropriate number of children for a family like yours?

RFPS - What are the appropriate number of children you would like to have?

\section{Future Contraceptive Intent.}

IFPS - Would you consider using some method to limit the number of children or to postpone a pregnancy in the future?

WFS - Would you or your husband consider using some me thod to limit number of children in the future?

RFPS - Do you have any possibility of using family planning methods in the future?

\section{Knowledge and Use of Methods.}

IFPS (a) Have you heard about any method of contraception?

(b) Did you ever use any method?

(c) What method did you use?

(d) Are you currently using any method?

WFS (a) Do you know or have you heard about any methods of contraception?

(b) What are the methods you know?

(c) Did you ever use any method?

(d) What methods?

(e) Are you using any method now? 
RFPS (a) Have you ever heard about family planning methods?

(b) What methods?

(c) Did you ever use contraceptives?

(d) What contraceptives did you use?

(e) Are you currently using any family-planning methods?

The independent variables included in the multivariate analysis can be classified into two broad categories: (1) demographic variables and (2) social variables. The demographic variables include age of respondents $(24+=1 ; 24$ or less $=0)$, age at marriage $(13+=1 ; 13$ or less $=0)$, and parity $(3+=1 ; 3$ or less $=0)$. These variables may reflect differential levels of "demographic pressure" as well as differentiate the target groups for family-planning programme.

The social variables include husband's years of education ( 1 year or more $=1$; none $=0)$, mother's years of education ( 1 year or more $=1$; none $=0)$, occupation (non-agriculture $=1$; agriculture $=0$ ), and the region (Dhaka and Chittagong $=1$; Rajshahi and Khulna $=0$ ). Level of development of Dhaka and Chittagong region is higher than those of Rajshahi and Khulna.

In Table 1, we present some descriptive statistics for currently-married women from our three sample surveys. Although the three sets of data are considerably different in sample size, the sample distribution by the categories of some selected variables are somewhat similar for all the three surveys. This result enhances our confidence in the quality of all the data sets and their comparability.

\section{AGE, PARITY, AND TRENDS IN KNOWLEDGE, USE; AND FUTURE INTENDED USE OF CONTRACEPTIVES AND IDEAL FAMILY SIZE}

Figure 1 and Table 2 depict the diffusion pattern of knowledge, current and ever uses, and future intended use of contraceptives by parity and age of respondents in rural Bangladesh for the years 1968 through 1977. In all the three surveys, data on the knowledge of family-planning methods were indicated by the respondents with or without being prompted by the interviewers. Figure 1 and Table 2 clearly show a steady increase in knowledge and practice of family planning over the years. Table 2 shows that knowledge of contraceptives among the respondents increased substantially from about 63 percent in 1968 to about 94 percent in 1977. Although current contraceptive use also increased from 3.6 percent in 1968 to 10.5 percent in 1977 , the actual practice of family-planning methods was less than the increased level of contraceptive knowledge. Similarly lagging family-planning response was evident in the Contraceptive Prevalence Survey which reported only 13 percent to be current users of contraceptives while 94.8 percent indicated having knowledge of contraceptives (Walliullah, 1981). Figure 1 further shows that a relatively higher rate of contraceptive use was found among those between age 25 and 34 as well as among those having higher number of children. 


\section{Table 1}

Descriptive Statistics for Currently-Married Women from the Three Samples: Impact of Family Planning Survey (IFPS) in 1968, World Fertility Survey (WFS) in 1975, and Rural Fertility and Poverty Survey (RFPS) in 1977

Variables

Years of Schooling IFPS WFS RFPS

Percent of Mothers having One or More Years of Schooling

20.0

Percent of Husbands doing Non-Agricultural Jobs

Mean Number of Children Ever Born

Average Age of First Marriage

Average of the proportion of children died after birth for the age-groups: 15-19, 20-24, $25-29,30-34,40-44$, and $45-49$.

\section{SOCIO-DEMOGRAPHIC FACTORS AND TRENDS IN KNOWLEDGE, USE, AND FUTURE INTENDED USE OF CONTRACEPTIVES AND IDEAL FAMILY SIZE: 1968-1977}

Table 2 also shows the percent distribution of contraceptive use, future contraceptive intent, knowledge of family-planning methods, and average family size by various social and demographic factors. The purpose of this analysis is to see the effects of selected social and demographic variables on the variation of these dependent variables. The data in the table show that in all the three periods -1968 , 1975, and 1977 - ever, current, and future intended uses and knowledge of contraceptives tended to be positively and moderately related to respondents' educational levels, non-agricultural occupation, and number of living children. The same was also true of educational levels of respondents' husbands. No consistent relationship was, however, found between these factors and ideal family size, although overall ideal family size had declined somewhat by the terminal year of 1977 , reflecting 


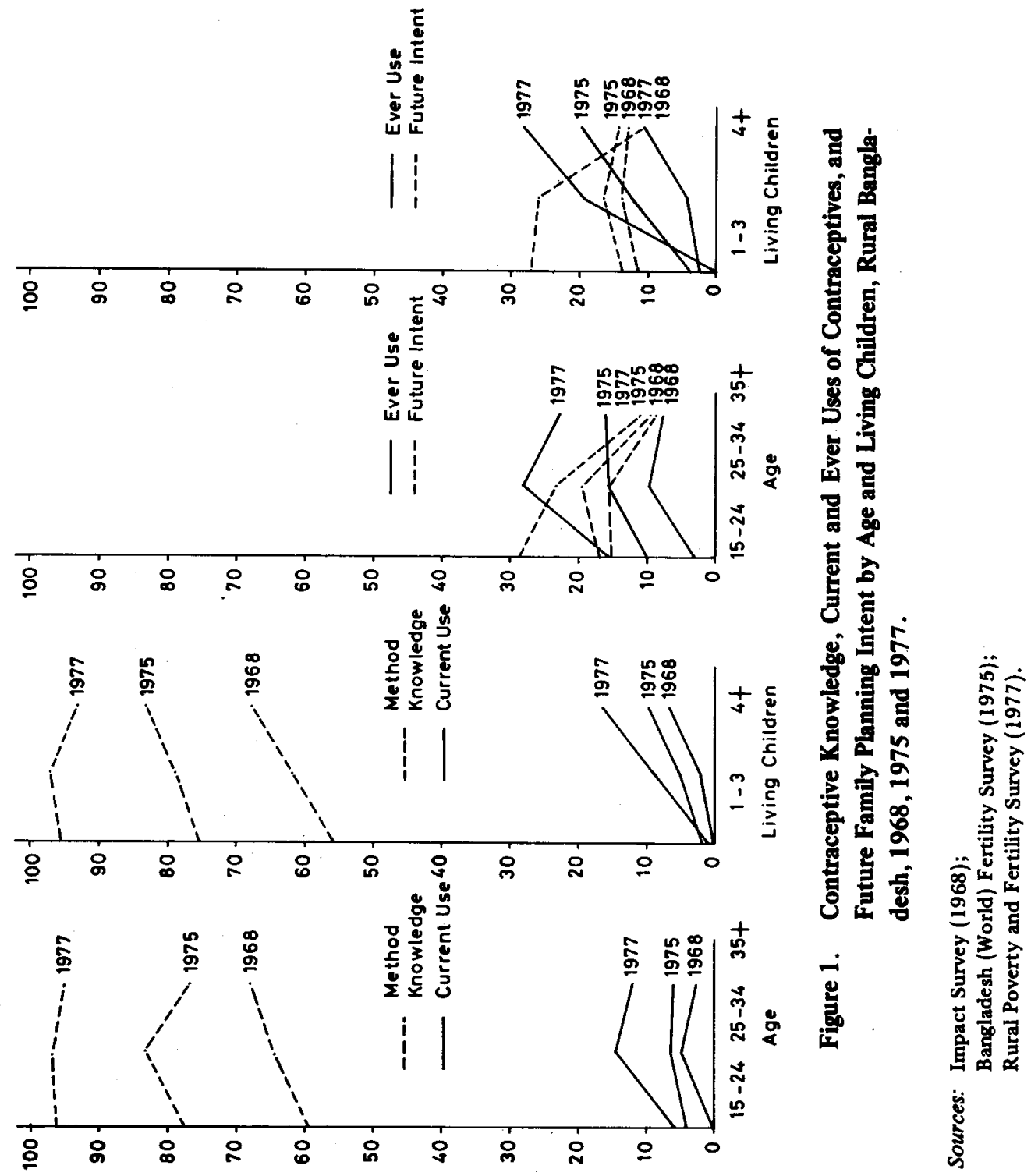


Table 2

Family Planning Knowledge, Ever and Current Uses of Contraceptives, Intention to Use Contraceptives, and Ideal Family Size by Various Characteristics of Rural Bangladesh Women, 1968-1977

\begin{tabular}{lccccccc}
\hline Variables & Year & $\begin{array}{c}\text { Ever } \\
\text { Used }\end{array}$ & $\begin{array}{c}\text { Currently } \\
\text { Using }\end{array}$ & $\begin{array}{c}\text { Intend } \\
\text { to Use }\end{array}$ & $\begin{array}{c}\text { Know F.P. } \\
\text { Method* }\end{array}$ & $\begin{array}{c}\text { Average } \\
\text { Ideal } \\
\text { Family Size }\end{array}$ & N** $^{* *}$ \\
\hline All: & 1968 & $6.0 \%$ & $3.6 \%$ & $13.3 \%$ & $62.6 \%$ & 4.4 (Means) & 2522 \\
& 1975 & 13.4 & 6.8 & 15.9 & 78.4 & 4.0 & 4117 \\
& 1977 & 19.3 & 10.5 & 17.7 & 94.5 & 3.3 & 1247
\end{tabular}

Husband's

Years of

Education

\begin{tabular}{rrrrrrrr}
0. & 1968 & 4.1 & 2.7 & 10.6 & 54.9 & 4.5 & 1446 \\
& 1975 & 10.9 & 5.6 & 14.8 & 73.3 & 4.0 & 2361 \\
& 1977 & 16.0 & 8.6 & 18.7 & 93.9 & 3.3 & 703 \\
& & & & & & & \\
\hline 1 & 1968 & 7.8 & 4.6 & 16.2 & 71.7 & 4.4 & 1076 \\
& 1975 & 16.8 & 8.3 & 17.4 & 85.3 & 3.9 & 1756 \\
& 1977 & 23.6 & 12.9 & 16.5 & 95.3 & 3.3 & 543
\end{tabular}

Mother's

Years of

Education

$\begin{array}{rrrrrrrr}0 & 1968 & 4.8 & 3.4 & 10.4 & 57.0 & 4.5 & 1930 \\ & 1975 & 11.0 & 5.4 & 15.2 & 73.5 & 3.8 & 3190 \\ & 1977 & 16.1 & 8.4 & 17.0 & 93.7 & 3.3 & 998 \\ & & & & & & & \\ & 1968 & 9.6 & 4.4 & 22.8 & 80.7 & 4.3 & 496 \\ & 1975 & 21.8 & 11.7 & 18.4 & 96.4 & 4.0 & 926 \\ & 1977 & 31.8 & 18.8 & 20.6 & 97.7 & 3.2 & 249\end{array}$

Age at

Marriage

\begin{tabular}{rrrrrrrr}
$\leqslant 13$ years & 196.8 & 5.3 & 3.1 & 12.2 & 61.8 & 4.5 & 1914 \\
& 1975 & 13.9 & 6.6 & 14.8 & 76.2 & 4.2 & 2445 \\
& 1977 & 18.1 & 8.6 & 15.3 & 94.2 & 3.3 & 606 \\
$>13$ years & 1968 & 7.9 & 5.3 & 16.0 & 65.0 & 4.3 & 608 \\
& 1975 & 11.8 & 5.7 & 14.2 & 81.5 & 3.9 & 1672 \\
& 1977 & 22.1 & 13.1 & 22.1 & 96.1 & 3.3 & 641 \\
\hline
\end{tabular}


Table 2 - (Continued)

Occupation:

Agricultural

$\begin{array}{rrrrrrr}1968 & 5.0 & 3.1 & 12.4 & 60.6 & 4.5 & 1194 \\ 1975 & 13.3 & 7.0 & 15.3 & 76.2 & 4.1 & 2322 \\ 1977 & 19.7 & 10.1 & 14.6 & 93.2 & 3.3 & 764\end{array}$

$\begin{array}{lllll}\text { Non- } & 1968 & 7.5 & 4.5 & 14.8\end{array}$

$65.7 \quad 4.3$

744

agricultural $1975 \quad 13.3 \quad 6.3 \quad 16.9$

$81.5 \quad 3.9$

1795

$\begin{array}{llll}1977 & 20.0 & 11.6 & 22.0\end{array}$

$96.7 \quad 3.3$

433

Mother's Age

$\begin{array}{rrrllllr}12-24 & 1968 & 2.8 \% & 1.2 \% & 15.2 \% & 59.0 \% & 4.2 & 1647 \\ & 1975 & 9.3 & 4.6 & 15.6 & 77.3 & 3.7 & 1781 \\ & 1977 & 15.2 & 7.0 & 24.7 & 95.2 & 3.1 & 420 \\ 24+ & 1968 & 8.1 & 5.3 & 12.1 & 65.1 & 4.7 & 875 \\ & 1975 & 16.1 & 7.5 & 13.9 & 78.9 & 4.3 & 2336 \\ & 1977 & 24.3 & 14.0 & 15.1 & 94.5 & 3.5 & 827\end{array}$

Number of

Living

Children

$\begin{array}{rrrrrrrr}0-3 & 1968 & 3.4 & 1.7 & 12.6 & 59.0 & 4.3 & 1488 \\ & 1975 & 9.7 & 4.6 & 13.9 & 76.3 & 3.8 & 2532 \\ & 1977 & 17.1 & 8.3 & 19.9 & 96.2 & 3.1 & 469 \\ & & & & & & & \\ >3 & 1968 & 9.7 & 6.4 & 14.3 & 67.9 & 4.5 & 1034 \\ & 1975 & 18.6 & 8.9 & 15.8 & 81.9 & 4.3 & 1585 \\ & 1977 & 23.1 & 13.2 & 13.4 & 94.1 & 3.4 & 778\end{array}$

Region:

$\begin{array}{rrrrrrrr}\text { Dhaka and } & 1968 & 6.6 & 4.3 & 15.4 & 62.6 & 4.4 & 1178 \\ \text { Chittagong } & 1975 & 10.1 & 5.0 & 14.0 & 82.3 & 4.3 & 1756 \\ & 1977 & 18.8 & 10.8 & 20.7 & 99.8 & 3.3 & 655\end{array}$

\begin{tabular}{crrrrrrr} 
Khulna and & 1968 & 5.4 & 3.1 & 11.5 & 62.6 & 4.4 & 1344 \\
Rajshahi & 1975 & 15.9 & 8.1 & 17.3 & 75.5 & 3.9 & 2361 \\
& 1977 & 19.9 & 10.2 & 14.4 & 90.0 & 3.3 & 590 \\
\hline
\end{tabular}

Sources: Impact Survey (1968).

Bangladesh (World) Fertility Survey (1975).

Rural Poverty and Fertility Survey (1977).

* Knowledge of at least one method based on prompted or unprompted response.

** Observations with missing data on any of the variables in the table are excluded from the analysis. 
fertility limitation measures. However, the effect of living children seemed to have decreased over the years as was evident in its net declining coefficient in the terminal year of 1977. This declining coefficient of living children suggested more increases in contraceptive use and future contraceptive intent among younger or lower-parity mothers. On the other hand, non-agricultural occupation tended to show no significant effect or showed slight positive effect on contraceptive use, while developed regions of Dhaka and Chittagong showed inconsistent effects. Similarly, age, age at marriage, and husband's education tended to show either inconsistent or insignificant relationship with ever, current, and future intended uses of contraceptives.

\section{SUMMARY AND DISCUSSION}

In the foregoing analysis, we have examined the response of Bangladeshi women to the demographic pressure and to the family-planning programme inputs during the period between 1968 and 1977. The findings clearly indicate that there had been a steady increase in knowledge, use, and future intended use of contraceptives between the years 1968 and 1977 . However, the absolute increase in the use of family-planning methods was less than the increase in the level of knowledge. While the increased level of knowledge and a moderate increase in the practice of familyplanning methods indicated the success of the expanding family-planning programme in reaching larger audiences, lag in actual practice compared with knowledge showed that mere awareness about family-planning method was insufficient for inducing its practice. The average ideal family size desire continued to be moderately high (ranging from 4.4 to 3.3) in Bangladesh, although over the years there had been a gradual shift toward smaller ideal family size. This, perhaps, reflected the beginning of the decline of a high-fertility culture because of vigorous campaigns for family planning. The increase in contraceptive use was, however, higher than that of future intent to use. This suggests that the expanding family-planning programmes were meeting more of the residual demand for fertility regulation than creating any new demand.

Analysis of contraceptive use and future contraceptive intent by parity for the periods 1968, 1975, and 1977 further indicated that the latter factor was important in inducing contraceptive use and future contraceptive intent. Over the years, the increase in contraceptive use and future contraceptive intent were found to be more marked among lower-parity mothers, indicating a trend towards a desire for small family size rather than responding to mere pressure from high parity in the choice of family size. The higher contraceptive use among high-fertility mothers indicates some success of the family-planning programme in converting population-pressureinduced latent demand into an overt one.

Examination of the effects of the various socio-economic factors, considered as proxies for modern influences, revealed mixed results. Contraceptive use and intent to use tended to be positively related to respondent's educational level, but weakly 
to non-agricultural occupations. Analysis of trend over time appeared to suggest no major socio-economic differences in the rate of change in contraceptive use, future contraceptive intent, or ideal family size desire. Similarly, no consistent pattern in the relationship of dependent variables with age at marriage or region was observed.

The detailed multivariate analysis by logit regression technique shows that the most important factors positively affecting family-planning acceptance and future family-planning intention were living children and respondent's educational level. However, the effect of living children had declined in magnitude over the years. While, over the years, the current use and intended future use had increased, the positive effect of living children indicated that demographic pressure had influenced this increase. On the other hand, some indicators of modern influences, such as developed region, non-agricultural occupation, or elevated age at marriage, showed inconsistent or insignificant relationships with contraceptive use. This, together with the relatively small coefficients of parity and education, suggests that none of these variables accounted for any major increase in family-planning demand.

The foregoing analysis shows that since there were no major socio-economic changes in Bangladesh, the recent moderate-rise in contraceptive adoption was probably the result of expansion of family-planning programmes. To the extent this was true, exposure to information, education, and communication campaigns for family planning promoted contraceptive adoption. However, the rise in contraceptive adoption was not large enough to constitute any significant family limitation response. There are a number of cultural and social factors in Bangladesh, such as low status of women, son preference, and female seclusion, that were likely to be responsible for such lack of significant response. Inadequate contraceptive supply and services may also have been another reason for this slacking response.

\section{REFERENCES}

Amin, R., and R. Faruqee. (1980). Fertility and Its Regulation in Bangladesh. Washington, D.C.: World Bank Staff Working Paper No. 383.

Amin, R., A. G. Mariam and Rashid Faruqee. (1985). "Fertility, Contraceptive Use, Socioeconomic Context and Fertility in Bangladesh". Demography India. Vol. 14. pp. 1-16.

Arthur, W. B., and G. McNicoll. (1978). "An Analytical Survey of Population and

Development in Bangladesh". Population and Development Review. Vol. 4. pp. 25-80.

Coale, A. (1973). "The Demographic Transition Reconsidered". Proceedings of International Population Conference, IUSSP. Liege.

Demeney, Paul. (1975). "Observations on Population Policy and Population Programme in Bangladesh". Population and Development Review. Vol. 1. pp. 307-322. 
Duncan, O. D. (1978). Minimum Logit Chi-square Regression using S.P.S.S. Department of Sociology, University of Arizona.

Entwisle, B., A. I. Hermalin, P. Kamnuansilpa, and A. Chamratrithirong. (1984). "A Multilevel Model of Family Planning Availability and Contraceptive Use in Rural Thailand”. Demography. Vol. 21. pp. 559-574.

Freedman, R. (1979). “Theories of Fertility Decline: A Reappraisal”. Social Forces. Vol. 58. pp. 1-17.

Freedman, R., and B. Berelson. (1976). "The Record of Family Planning Programmes". Studies in Family Planning. Vol. 7. pp. 1-40.

Goodman, L. A. (1972). "A Modified Multiple Regression Approach to the Analysis of Dichotomous Variables". American Sociological Review. Vol. 37. pp. 28-46.

Hankushek, E. A., and J. E. Jackson. (1977). Statistical Methods for Social Scientists. New York: Academic Press.

Kirk, D. (1972). “A New Demographic Transition?" In National Academy of Sciences, Rapid Population Growth.

Knodel, J. (1977). "Family Limitation and the Fertility Transition: Evidence from the Age Patterns of Fertility in Europe and Asia". PPopulation Studies. Vol. 31.pp. 219-249.

Knodel, J., and N. Debavalya. (1978). “Thailand's Reproductive Revolution". International Family Planning Perspectives and Digest. Vol. 4. pp. 34-49.

Knodel, J., N. Havanon and P. Pramualratana. (1984). "Fertility Transition in Thailand". Population and Development Review. Vol. 11. pp. 297-328.

Knoke, D., and P. J. Burke (1980). "Log-linear Models". Sage University Paper.

Mauldin, W. P., and B. Berelson. (1978). "Conditions of Fertility Decline in Developing Countries, 1965-1975". Studies in Family Planning. Vol. 9. pp. 90-147.

Raventholt, R. T., and R. T. Chao. (1974). "Availability of Family Planning Services: The Keys to Rapid Fertility Reduction". Family Planning Perspectives. Vol. 6. pp. 217-223.

Sirageldin, I., D. Norris and M. Ahmad. (1975a). "Fertility in Bangladesh: Facts and Fancies". Population Studies. Vol. 29. pp. 207-215.

Sirageldin, I., M. Hossain and M. Cain. (1975b). "Family Planning in Bangladesh: An Empirical Investigation". Bangladesh Development Studies. Vol. 3. pp. 1-26.

Teachman, J., D. J. Bogue, J. Londono and D. Hogan (1979). The Impact of Family Planning Programmes on Fertility Rates. Chicago: University of Chicago Community and Family Study Centre.

Tsui, A. O., and D. J. Bogue. (1978). "Declining World Fertility: Trends, Causes, Implications". Population Bulletin. Vol. 33.pp. 231-260.

Walliullah, S. (1981). Country Paper: Bangladesh. Asian Regional Contraceptive Prevalence Survey, Bangkok.

Zaidi, S. A. H. (1970). The Village Culture in Transition: A Study of East Pakistan Rural Society. Honolulu: East-West Press. 